ARTICLE

DOI: $10.1038 / s 41467-017-01118-x$

\title{
Genome-wide genetic and epigenetic analyses of pancreatic acinar cell carcinomas reveal aberrations in genome stability
}

Cornelia Jäkel ${ }^{1}$, Frank Bergmann², Reka Toth¹, Yassen Assenov¹, Daniel van der Duin', Oliver Strobel ${ }^{3}$, Thomas Hank $^{3}$, Günter Klöppel ${ }^{4}$, Craig Dorrell (1) ${ }^{5}$, Markus Grompe (1) ${ }^{5}$, Joshua Moss ${ }^{6}$, Yuval Dor ${ }^{6}$, Peter Schirmacher ${ }^{2}$, Christoph Plass ${ }^{1}$, Odilia Popanda ${ }^{1} \&$ Peter Schmezer ${ }^{1}$

Pancreatic acinar cell carcinoma (ACC) is an aggressive exocrine tumor with largely unknown biology. Here, to identify potential targets for personalized treatment, we perform integrative genome-wide and epigenome-wide analyses. The results show frequently aberrant DNA methylation, abundant chromosomal amplifications and deletions, and mutational signatures suggesting defective DNA repair. In contrast to pancreatic ductal adenocarcinoma, no recurrent point mutations are detected. The tumor suppressors ID3, ARIDIA, APC, and CDKN2A are frequently impaired also on the protein level and thus potentially affect ACC tumorigenesis. Consequently, this work identifies promising therapeutic targets in ACC for drugs recently approved for precision cancer therapy.

\footnotetext{
${ }^{1}$ Division of Epigenomics and Cancer Risk Factors, German Cancer Research Center (DKFZ), Im Neuenheimer Feld 280, 69120 Heidelberg, Germany. ${ }^{2}$ Institute of Pathology, University Hospital Heidelberg, Im Neuenheimer Feld 224, 69120 Heidelberg, Germany. ${ }^{3}$ Department of General and Visceral Surgery, University Hospital Heidelberg, Im Neuenheimer Feld 110, 69120 Heidelberg, Germany. ${ }^{4}$ Institute of Pathology, Technical University Munich, Trogerstr. 18, 81675 Munich, Germany. ${ }^{5}$ Department of Pediatrics, Papé Family Pediatric Research Institute, Oregon Stem Cell Center, Oregon Health and Science University, Portland, OR 97239, USA. ${ }^{6}$ Department of Developmental Biology and Cancer Research, Institute for Medical Research Israel-Canada, The Hebrew University-Hadassah Medical School, 9112102 Jerusalem, Israel. Correspondence and requests for materials should be addressed to P.S. (email: p.schmezer@dkfz.de)
} 
$\mathrm{P}$ ancreatic acinar cell carcinomas (ACC) are aggressive cancers that most frequently affect patients in their sixth and seventh decades of life, but may occur at any age, including childhood. At the time of diagnosis, $\sim 50 \%$ of ACC present with metastases ${ }^{1-4}$. Phenotypically, ACC resemble non-neoplastic acinar cells ${ }^{1}$. Rarely, ACC display a substantial neuroendocrine mixed differentiation, then being designated as mixed acinar-neuroendocrine carcinoma (MACNEC) ${ }^{1}$. Apart from tumor resection, which is the first line therapy in localized disease, therapeutic options in advanced disease are limited and have shown varying results ${ }^{5}$. New treatment strategies, including targeted drugs, are therefore needed. This is hampered by the fact that the molecular background of ACC is still only poorly understood, in part because it is a rare pancreatic neoplasm that accounts for $<2 \%$ of all pancreatic cancers ${ }^{4}$. This is in contrast to (i) pancreatic ductal adenocarcinomas (PDAC) accounting for over $90 \%$ of pancreatic cancers, where molecular alterations, e.g., in KRAS, TP53, or CDKN2A are well known ${ }^{6,7}$ and (ii) pancreatic neuroendocrine tumors (PNET) that show frequent alterations, e.g., in MEN1, MUTYH, or CHEK2 ${ }^{8}$. Several smaller studies revealed that these genes are only rarely affected in $\mathrm{ACC}^{9-12}$. Two recent genome-wide exome-sequencing studies demonstrated point mutations in $\mathrm{ACC}^{13,14}$, although no highly recurrent point mutations were detected, and the most common point mutation occurred in only $25 \%$ of the tumors in the SMAD4 gene $\mathrm{e}^{13,14}$. In a study using comparative genomic hybridization analysis, numerous chromosomal imbalances were detected ${ }^{15}$. Albeit, resolution of this array-based study is very limited thus making it difficult to pinpoint distinct important driver aberrations. Hence, no frequently recurrent point mutations or genetic driver events were found in ACC, suggesting other predominant mechanisms of tumor development.

Additional support for this assumption comes from findings that the Wnt pathway is affected in at least a subset of ACC. Mutations in APC have been reported in about $10-20 \%$ of cases $^{9}, 11,14$ and activation of the CTNNB1 protein has been reported in about $12-15 \% 9,16$. One study identified higher recurrence of aberrations by investigating APC on the level of mutations, deletions, and hypermethylation, and therefore detected changes in 7,48 , and $56 \%$ of cases, respectively ${ }^{12}$. However, data on the APC protein expression are still lacking.

In this study, we investigate two independent cohorts with a total number of 73 ACC and 34 normal pancreatic tissue samples representing one of the largest available tissue-based collectives world-wide. We aimed to examine the molecular aberrations in ACC in a comprehensive manner by investigating point mutations, DNA methylation, and copy number alterations employing whole-exome sequencing (WES) and Illumina's $450 \mathrm{~K}$ array (450K). In summary, we show that ACC do not display recurrent point mutations, but exhibit distinct mutational signatures. They harbor a large number of gene deletions and differential methylation of promoter sites and genes. This may provide directions for targeted therapeutic interventions.

\section{Results}

Genetic profiles confirm no recurrent point mutations in ACC. To investigate molecular alterations in ACC, whole exomes of ACC with available matching normal tissue were sequenced (22 ACC from cohort 1; see Supplementary Table 1). We obtained a median number of 137 point mutations per tumor with a range from 40 to 1023 mutations per tumor (Fig. 1a), similar to previous reports by Jiao et al. ${ }^{14}$ and Furukawa et al. ${ }^{13}$ (Fig. 1b, c). The high mutational load ( $>1000$ mutations) of one tumor was explained by microsatellite instability of this tumor, previously reported in Bergmann et al. ${ }^{15}$ (Supplementary Data
File 1). Genes mutated in $23 \%$ of carcinomas were COL $12 A 1$, $F R Y, F R Y L$, and PLB1 followed by CACNA1A, CCDC57, COL23A1, MKL2, RAP1GAP, SMAD4, and SRCAP occurring in $18 \%$ of carcinomas (Fig. 1b, for a detailed list of point mutations refer to Supplementary Data File 1). Genes that are often mutated in PDAC were either not mutated in our or published ACC datasets, e.g., KRAS, or were rarely mutated in published (3 out of 26) or our datasets ( 2 out of 22 ), e.g., TP53 ${ }^{6,7}$. After employing MutSigCV ${ }^{17}$, which identifies genes that are significantly mutated, no gene remained significantly enriched. Overall, no recurrent point mutations were identified in ACC suggesting that point mutations play a minor role in ACC.

Mutational signatures identify risk factors for ACC. To identify potential factors for tumor development, we calculated mutational signatures using the WES data. Different carcinogens such as UV light or tobacco have specific impacts on the mutational patterns of cancer ${ }^{17}$. Calculation of the mutational frequency of point mutations (i.e., $\mathrm{C}>\mathrm{A}, \mathrm{C}>\mathrm{G}, \mathrm{C}>\mathrm{T}, \mathrm{T}>\mathrm{A}, \mathrm{T}>\mathrm{C}, \mathrm{T}>\mathrm{G}$ ) in the context of one base upstream and one base downstream identified frequent $\mathrm{C}>\mathrm{A}$ and $\mathrm{C}>\mathrm{T}$ point mutations in ACC (Fig. 1d), which is similar to observations in other tumor entities (Supplementary Fig. 1a). Comparing these signatures to published mutational signatures as depicted in the COSMIC database using the deconstructSigs package $^{18}$ (Fig. 1e; Supplementary Fig. 1b), 18 out of the previously published 30 signatures were identified in ACC. Each tumor displayed on average 4 signatures (range: 1-6; for details refer to Supplementary Data File 2). Signature 1, corresponding to deamination of 5-methylcytosine is found in most cancer types. This mutational signature was found in 20 out of 22 ACC. Signature 4, which is related to tobacco smoking and was not observed in PDAC ${ }^{19}$, was present in 12 tumors. In combination with tobacco chewing (signature 29), which was detectable in two patients, almost two-third (14 of 22) of ACC had mutational signatures associated with tobacco-associated carcinogens. Signatures associated with defective DNA repair (signatures 3, 6, 15, and 20) were identified in 15 of 22 tumors. Signature 3 is specifically associated with BRCA1 and BRCA2 mutations, whereas the other three are associated with defective DNA mismatch repair. Thus, ACC revealed distinct mutational signatures. This suggests defects in DNA double-strand break and DNA mismatch repair, which may contribute to the high genomic instability observed in this disease (see below).

DNA methylation profiles suggest cell of origin. To obtain a global picture of DNA methylation patterns and to identify copy number aberrations (CNA), we subjected 22 primary tumors, 12 metastases and 20 normal tissues from cohort 1 and 19 primary tumors and 10 normal tissues from cohort 2 to $450 \mathrm{~K}$ analysis (for an overview refer to Supplementary Table 1). First, we assessed the similarity and quality of the two cohorts. (i) Cluster analysis of the two cohorts revealed that their global methylation pattern is very similar and comparable (Supplementary Fig. 2d). (ii) Very high tumor content achieved by microdissection by a pathologist was validated via the tumor purity estimation package $\mathrm{LUMP}^{20}$ in 80 out of 83 samples (Supplementary Methods, Supplementary Fig. 2c). Principal component analysis (PCA) of all CpG sites of both cohorts revealed that normal pancreatic tissues clustered very closely together, whereas the tumors were distinct from the normal tissues and formed a wide-spread cluster, presenting a different global methylation pattern (Fig. 2a). To compare ACC to other pancreatic tumors, we further generated $450 \mathrm{~K}$ data for 17 pancreatic neuroendocrine tumors (PNET) and used data for 146 PDAC generated by The Cancer Genome Atlas (TCGA) ${ }^{21}$. A DNA methylation phylogenetic tree separated the three 
a

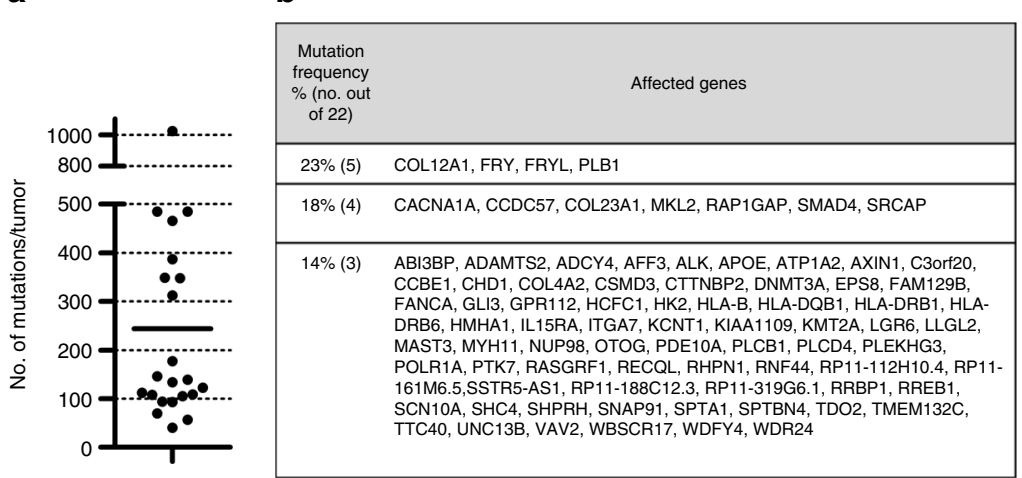

d

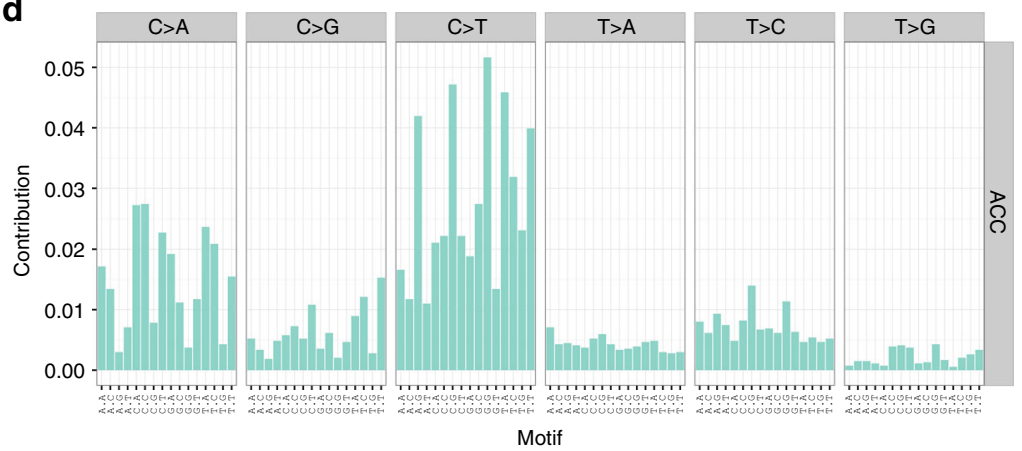

C
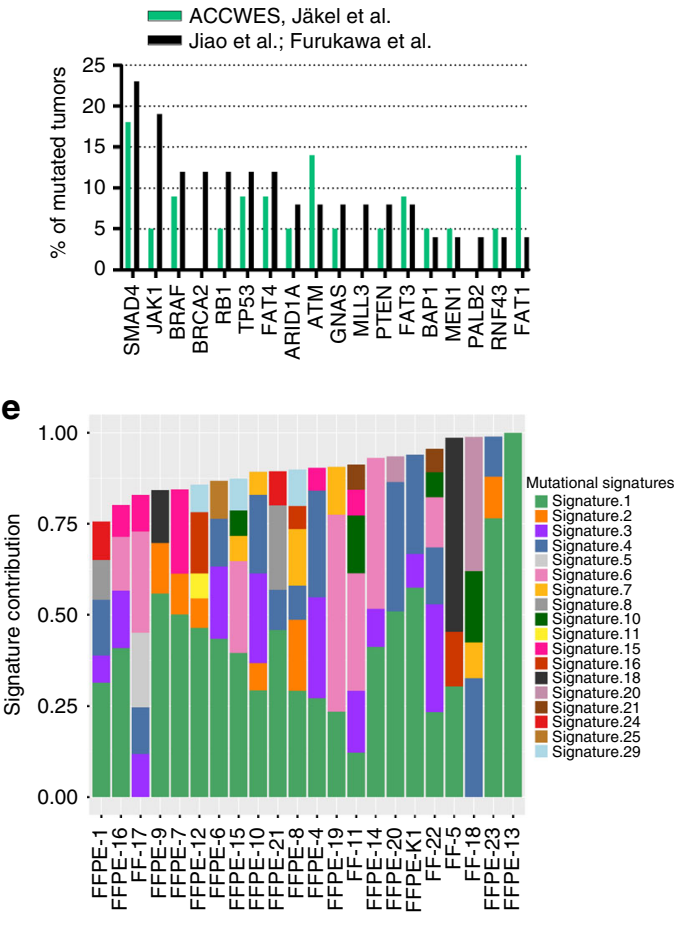

Fig. 1 ACC display a high-mutational load and specific mutational signatures. a Number of mutated genes per tumor detected by WES. b Genes with most recurrent, however, still low frequency point mutations in ACC. c Comparison of WES results to published studies ${ }^{12,13}$ of ACC shows a similar low frequency of recurrent mutations. Both published studies ${ }^{12,13}$ were utilized as a comparison in combination as one of them only contained three ACC. d Mutational pattern of ACC. The type of base substitution in their genomic context (base preceding and base following) is displayed. e Published mutational signatures ${ }^{27}$ present in each sample is displayed. Associations of signatures: signature 1: deamination of 5-methylcytosine, signature 4: tobacco smoking, signature 29: tobacco chewing, signature 3: DNA double-strand break repair defects, signature 6: defective DNA mismatch repair and microsatellite unstable, signature 15: defective DNA mismatch repair, signature 20: defective DNA mismatch repair

included pancreatic cancer types based on their methylome (Fig. 2b). As the cell of origin is unknown for ACC, we employed the algorithm of Houseman et al. ${ }^{22}$ to infer the contribution of DNA methylation profiles of sorted normal pancreatic cell types (acinar, duct, neuroendocrine $\alpha$-cells, and $\beta$-cells) to the methylation profiles of the pancreatic tumor types. The analysis revealed high similarities between the methylation profiles of (i) ACC and acinar cells (ii) PNET and $\alpha$-cells, and (iii) PDAC and duct cells but also to some extent acinar cells (Supplementary Fig. 2a). Therefore, ACC should ideally be compared to healthy acinar cells for identifying differentially methylated regions. As formalin-fixed, paraffin-embedded (FFPE) tissues were used for $450 \mathrm{~K}$ analysis and sorted acinar cells from adjacent tissue were not available, we used adjacent pancreatic tissue rich in acinar cells ( $90 \%$ of cells) as reference for comparing tumors versus normal tissues.

We detected frequent and recurrent hypermethylation at 44,193 CpG sites in cohort 1 (Fig. 2c) and 26,959 sites in cohort 2 (Supplementary Fig. 2b). After mapping the CpG sites to either promoter sites, $\mathrm{CpG}$ islands, the overlap of $\mathrm{CpG}$ islands and promoters ( $\mathrm{CpG}$ island promoters) or gene bodies (for details refer to "Methods"), we obtained differentially methylated regions in cohort 1 in a total number of 466, 690, 245, and 608 regions, respectively. Between 44 and $77 \%$ of these regions were validated in cohort 2 , resulting in a final number of $245,411,189$, and 270 regions differentially methylated in both cohorts (Fig. 2d, e, for beta values and differential methylation calling refer to Supplementary Data Files 3-5. Hypermethylation was predominant in these regions and only about one quarter of differentially methylated regions in promoter $\mathrm{CpG}$ islands and genes were hypomethylated (Fig. 2d). A total number of 512 genes with differential methylation at their promoter site and/or gene body were identified (Supplementary Data File 6) in cohort 1 and validated in cohort 2 . Gene ontology enrichment analysis of these differentially methylated regions was performed and revealed enrichment of developmental and cell adhesion pathways (Fig. 2f). The homophilic cell adhesion pathway including the protocadherins was the most enriched pathway (Supplementary Fig. 2e). Protocadherins are clustered in a $700 \mathrm{~kb}$ genomic region and hypermethylation of six promoters in this region was confirmed via MassARRAY (Supplementary Methods, Supplementary Fig. 2f).

Next, we investigated whether methylation changes upon metastases formation can be detected. Primary tumors always clustered close to their metastases (Supplementary Fig. 2c) and paired differential methylation analysis revealed only few differences, indicating that the global methylation profile remained stable in metastases (Supplementary Fig. 2d, e).

In summary, we found that (i) the methylation profile of ACC corresponds to non-neoplastic acinar cells, (ii) ACC display considerable differential methylation, and (iii) methylation aberrations of ACC metastases are comparable to their primary tumors.

ACC harbor many chromosomal gains and losses. $450 \mathrm{~K}$ array signal intensities can be used to generate CNA maps ${ }^{23}$. Strikingly, ACC harbored many deletions and amplifications (Fig. 3a, cohort 
1; Supplementary Fig. 3a, cohort 2), in particular a broad range loss of chromosome arm 1p (especially 1p36) and gain of chromosome 1q (especially 1q42). Localized highly recurrent deletions in 9 p21.3, 16p13.3, and 18q21.2 loci and amplifications in 7p22.3 were also identified. In total, 2324 and 323 genes mapped to the deleted and amplified regions, respectively (only taking regions into account where not the whole chromosome arm was altered; Supplementary Data Files 7 and 8). About $62 \%$ of deleted and $11 \%$ of amplified genes were confirmed with the $450 \mathrm{~K}$ data of cohort 2, resulting in 1441 and 35 confirmed deleted and amplified genes (Fig. 3b, c; Supplementary Data File 7). Four of these regions were additionally confirmed by qPCR (Supplementary Methods, Supplementary Fig. 5b). Thus, these large numbers of recurrent CNA were indicative of a high chromosomal instability in ACC. This instability did not increase when primary tumors were compared with their corresponding metastases (Supplementary Fig. 3b), similar to observations in $\mathrm{PDAC}^{24}$.

Integrative analysis identifies frequently altered genes. We next integrated our data on DNA methylation and CNA. Circos plots showed that the hypermethylated and hypomethylated regions were quite evenly distributed throughout the genome, whereas the amplified and deleted regions tended to be localized (Fig. 4a; Supplementary Fig. 4). Each gene in each tumor was subsequently put into one of nine categories depending on whether the gene in that tumor was hypomethylated or hypermethylated at its promoter region, and/or amplified or deleted, or unaltered. This integrative approach revealed that previously not reported, frequent aberrations could be identified in ACC of which the 100 most affected genes are depicted in Fig. 4b. We then classified these aberrations using published reference lists ${ }^{25-28}$ into the previously defined categories from these publications (known cancer genes, candidate cancer genes, and oncomirs from King's college $^{28}$; tumor suppressors from Vanderbuilt; ${ }^{27}$ mutated driver genes, driver genes CNA, and cancer predisposition genes from Vogelstein et al.; ${ }^{26}$ epigenetic regulators from Plass et al. ${ }^{25}$ ). Using this approach, we discovered 292 genes significantly altered in ACC, which are already known to play a role in tumorigenesis in other cancer entities (Supplementary Data File 9).

Specific tumor suppressors are frequently lost in ACC. We next investigated whether the observed epigenetic and genetic aberrations in ACC are associated with a change of the respective protein expression. Immunohistochemical stainings were performed on a tissue microarray including 23 ACC from cohort 1 and 39 ACC from cohort 2 as well as 8 normal pancreatic samples. The protein expression of 8 aberrant genes was evaluated: ARID1A, APC, CDKN2A, HIST1H, ID3, JAK1, PCDHG, and SOX2. This selection of candidate genes from our integrative analysis results list was necessary for practical reasons such as experimental capacity and availability of high quality antibodies. There are however more potential cancer driver genes in our list, which could not be further evaluated within the presented study. a

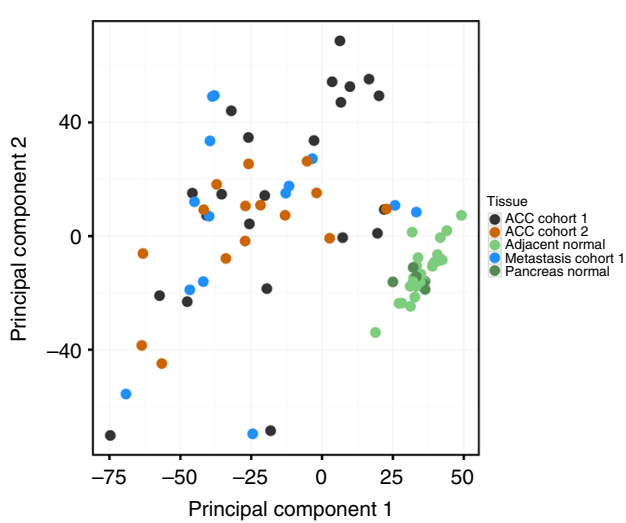

C

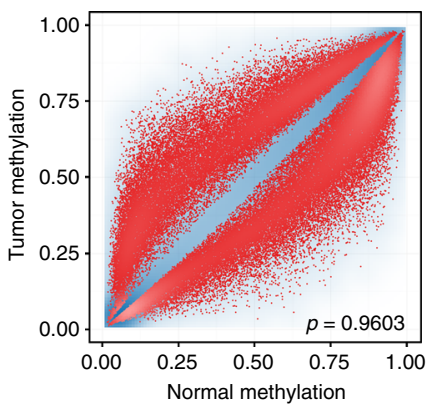

d

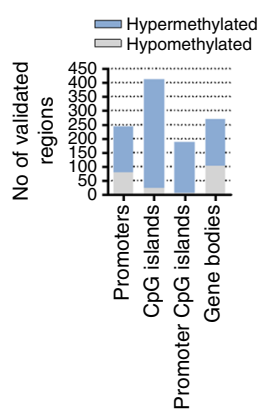

b

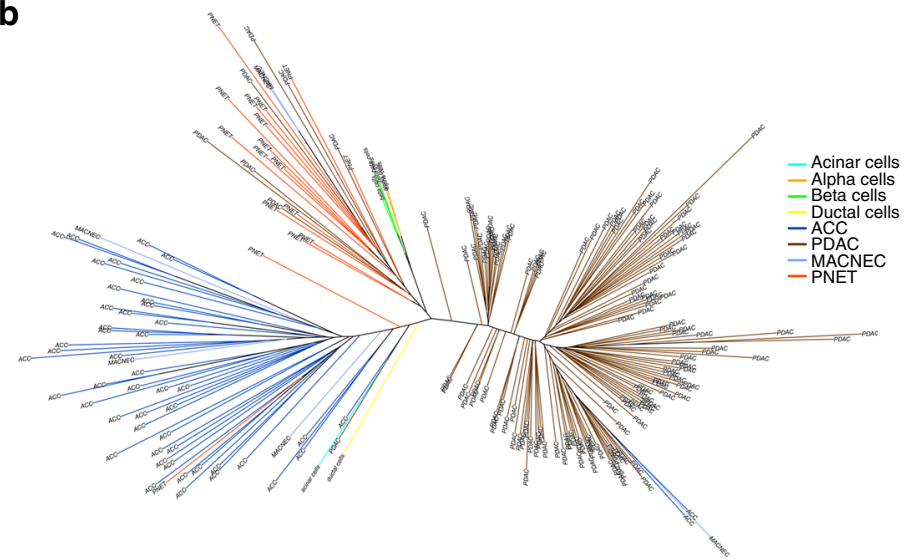

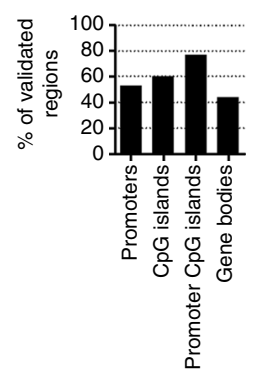

f

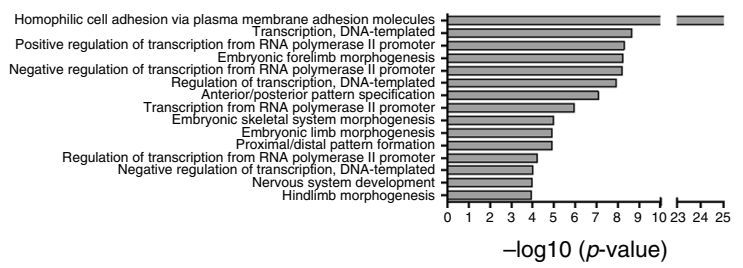

Fig. 2 ACC display a unique methylome and many recurrent differentially methylated regions. a Principal component analysis of all CpG sites of cohort 1 and cohort 2. b DNA methylation phylogenetic tree, including ACC, MACNEC, PNET, PDAC, and sorted pancreatic cells. c Methylation scatterplot of all CpG sites on the $450 \mathrm{~K}$ array in cohort 1 reveal massive differential methylation comparing all primary tumors versus all normal tissues. (red: sites above the rank cutoff, i.e., considered significant, blue: transparency corresponds to density of sites.). $\mathbf{d}$ Differential methylation defined by the rank cutoff in regulatory regions identified in cohort 1 and validated in cohort 2. e Validation rate of regions identified in cohort 1 and validated in cohort 2. $\mathbf{f}$ GO enrichment analysis displaying enriched pathways of the differentially methylated regions (input: regions of cohort 1 confirmed in cohort 2) 
Four of these genes (ARID1A, APC, CDKN2A, and ID3) showed significantly reduced protein expression in the majority of tumor samples (Fig. 5a), whereas the remaining four genes displayed changes only in a few tumors (Supplementary Fig. 5a). ID3 was downregulated in 89 and 94\%, ARID1A in 68 and 74\%, APC in 71 and $62 \%$, and CDKN2A in 53 and $52 \%$ of samples from cohort 1 and 2, respectively (Fig. 5b). Strikingly, most tumors displayed protein alterations in more than one of these four tumor suppressor genes (Fig. 5c). Nineteen tumors had alterations in all 4 genes, 21 tumors in 3 genes, and 13 tumors in 2 genes (Fig. 5d). Interestingly, ID3 and ARID1A alterations were evident in 60 out of 61 tumors, suggesting that downregulation of these two tumors suppressors are highly important. It was possible to predict the majority of protein losses based on the aberrant DNA methylation and CNA (Fig. 5e). Molecular alterations were additionally confirmed with independent methods (Supplementary Fig. 5b). As expected from the WES results, point mutations did not add any additional value to this. Taken together, we identified losses of several tumor suppressor genes which affect a majority of ACC suggesting an important role of these genes during the initiation and progression of these tumors.

\section{Discussion}

In the present study, we investigated molecular aberrations in ACC on a genome-wide scale and on multiple levels. In recent years, ACC which represent a distinct tumor entity, that morphologically and immunohistochemically differ from PDAC or PNET, were shown to typically lack driver mutations known from the more common ductal carcinogenesis ${ }^{6-8,29}$. We confirm that ACC do not display highly recurrent point mutations. However, our results show that ACC have highly unstable tumor genomes with localized, but also broad-ranged, chromosomal gains and losses. In addition, ACC exhibit mutational signatures that are associated with tobacco consumption and defective DNA repair. Common target genes clearly differ between ACC and other pancreatic tumors. For instance, ACC, but not PDAC or PNET harbor frequent aberrations in APC $(60-70 \%$ versus $<20 \%)^{8,30,31}$, and exhibit a loss of ID3, which in contrast appears to be overexpressed in PDAC ${ }^{32,33}$. Previously it has been shown that the tumors' methylation profiles resemble that of their cell of origin $^{34-36}$, thus one has to be careful when calculating differential methylation to actually identify cancer-related methylation changes. To that end, we used methylation profiles from sorted pancreatic cells. Morphologically, ACC resemble non-neoplastic

a
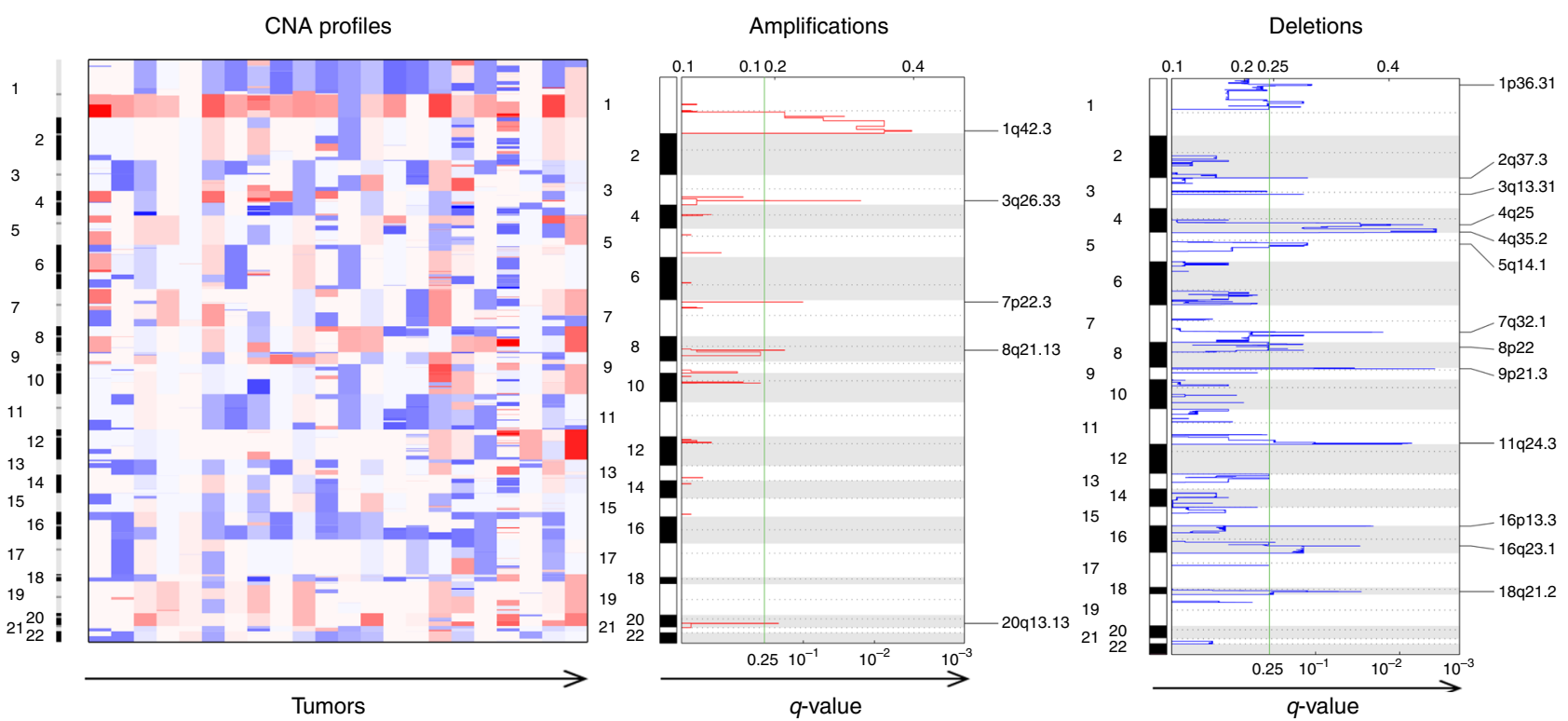

b

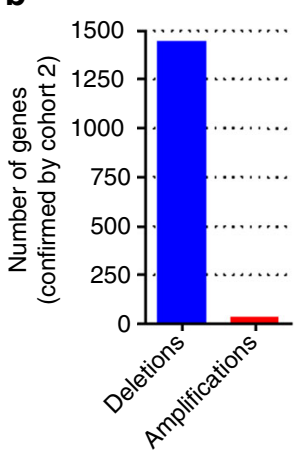

C

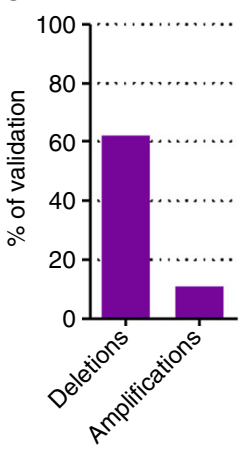

d

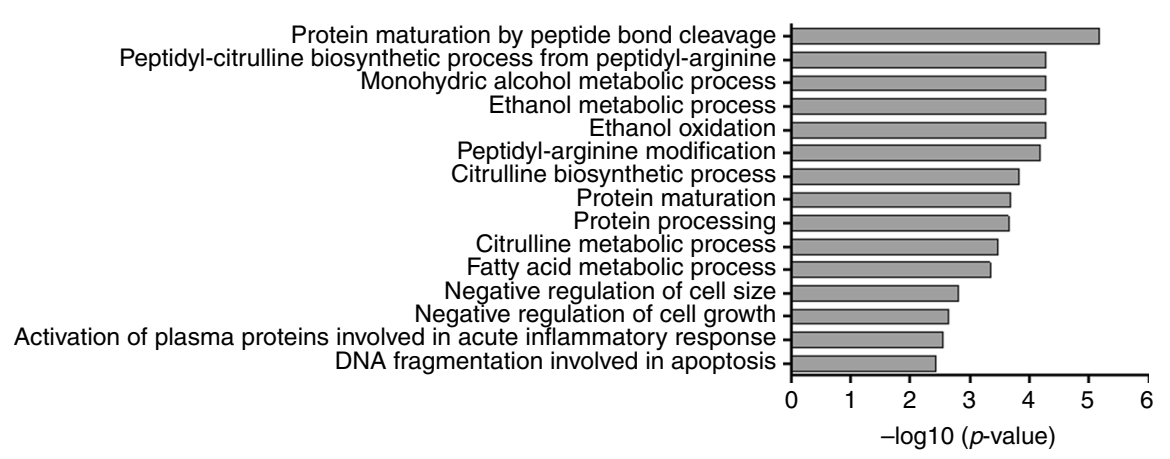

Fig. 3 ACC are characterized by numerous copy number alterations, mainly deletions. a CNA profiles: heatmap of copy numbers calculated via the intensities of the 450K array (each tumor versus average normal) are displayed for each tumor of cohort 1 and each chromosome (red: amplifications, blue: deletions). Amplifications: $q$ values of amplifications of all tumors of cohort 1. Deletions: $q$ values of deletions of all tumors of cohort 1. $\mathbf{b}$ Confirmed number of genes mapping to significantly amplified or deleted regions. c Percentage of genes significantly amplified or deleted of cohort 1 that were confirmed in cohort 2. d GO enrichment analysis displaying enriched pathways of amplified and deleted genes (input: regions of cohort 1 confirmed in cohort 2) 
a

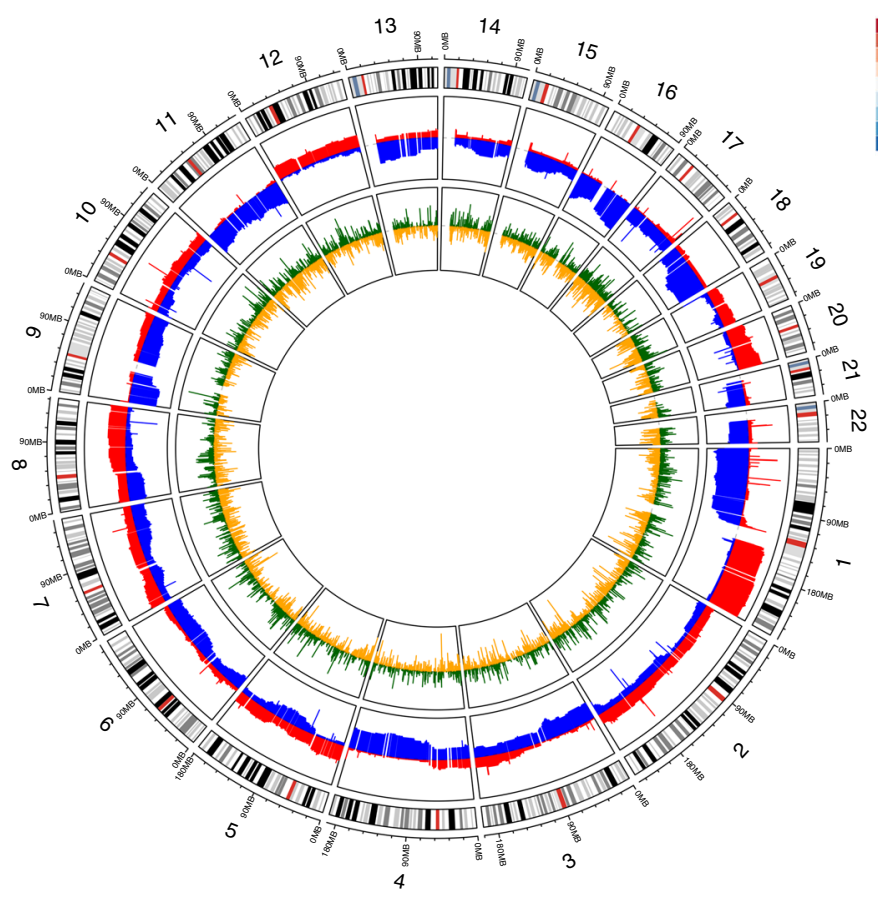

b

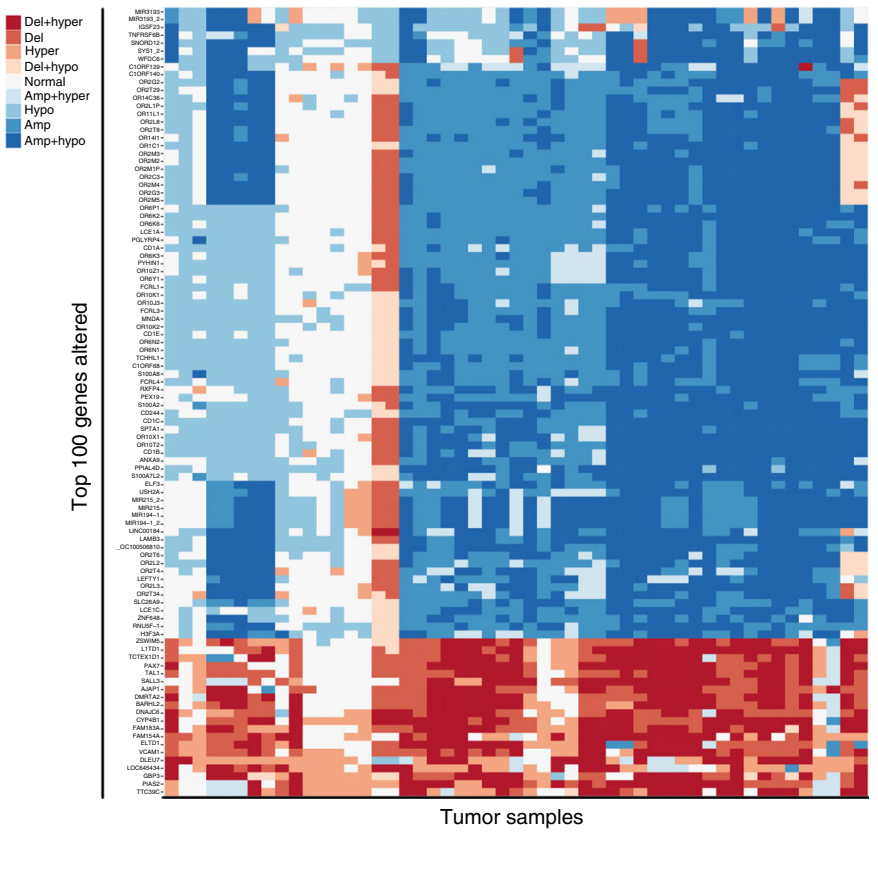

Fig. 4 Frequent molecular alterations can be identified in ACC. a Circos plot displaying all autosomes (outer circle, grey, centrosomes red) with amplifications (middle circle, red), deletions (middle circle, blue), hypermethylation (inner circle, green), hypomethylation (inner circle, yellow). b Heatmap of top 100 genes encompassing copy number aberrations and differential promoter methylation; red: leading to loss of gene function, blue: leading to gain of gene function. The darker the color the more aberrations were detected

acinar cells ${ }^{37}$, and this relation could also be reproduced on the molecular level, as ACC revealed a similar methylation profile to acinar cells. A further finding based on the methylation profiles of previously published sorted blood cells ${ }^{22}$ is that ACC contain only few infiltrating immune cells, consisting mainly of CD8+ T-cells, which still has to be confirmed via immunohistochemistry (IHC). In contrast to that, PDAC are infiltrated by many immune cells with CD4+ T-cells prevailing (Supplementary Fig. 2a), as previously shown via $\mathrm{IHC}^{38}$. The molecular profile of MACNEC did not differ from pure ACC, suggesting a common molecular background despite morphological differences. However, we identified intertumoral differences based on the molecular aberrations in ACC. We were not able to explain these subgroups by clinical or molecular parameters, though (Supplementary Fig. 6). All these data further support the notion that these pancreatic cancers are distinct not only on the clinical and pathological but also profoundly different on the molecular level.

We used $450 \mathrm{~K}$ arrays for copy number analysis, which enables its evaluation in parallel to DNA methylation analysis from the same DNA specimen ${ }^{23}$. It has been proven to be as robust and sensitive as SNP arrays. Although it may be less adequate than SNP arrays to detect alterations in large intergenic regions or gene desert regions, Feber et al. ${ }^{39}$ showed that it provides good coverage of the majority of coding loci. ACC are characterized by an imbalanced genome with numerous chromosomal gains and losses (Fig. 3). This chromosomal instability may be explained by (i) loss of ARID1A, BRCA1/2 ${ }^{13}$, and CENPE (Supplementary Data File 9), and (ii) mutational signatures associated with defective DNA repair mechanisms. ARID1A protein is lost in $\sim 70 \%$ of the tumors. As part of the SWI/SNF complex, it is involved in non-homologous end joining ${ }^{40}$, maintains proper chromosome segregation and prevents anaphase bridges during mitosis ${ }^{41}$. Its loss might contribute to the massive chromosomal gains and losses observed in ACC. BRCA2 is also important in maintaining genomic stability because of its involvement in homologous recombination. Furukawa et al. ${ }^{13}$ reported BRCA2 point mutation in 3 out of 7 ACC and a downregulation at the protein level in 5 out of 11 ACC. Others have reported lower BRCA2 mutation frequencies ${ }^{2}$. In our series, about $70 \%$ of the ACCs had mutational signatures associated with DNA repair defects. The mutational signature 3 , which is thought to be caused by BRCA1 and/or BRCA2 loss, can be detected in 10 out of the 22 sequenced tumors. Signatures that are associated with DNA mismatch repair occured in 11 tumors. These data reflect the important role that defective DNA repair pathways may have in ACC tumorigenesis.

In addition, cell cycle control is impaired in ACC by frequent abrogation of APC, CDKN2A, and ID3, which are all negative regulators of the cell cycle (Fig. 6). APC inhibits CTNNB1, which in turn activates the transcription factors (TCFs). One of the target genes of TCFs is cyclin D (CCND), which is a regulator of cell cycle progression ${ }^{42,43}$ In ACC, the cell cycle inhibitor CDKN2A was so far not reported to be frequently affected ${ }^{12,44}$, mostly due to the fact that in these studies only mutations had been investigated. Here, we report an absence of CDKN2A protein expression in $>50 \%$ of cases and an overexpression in 8 cases suggesting alterations within the $\mathrm{pRb}$ pathway ${ }^{45}$. Nearly, all tumors $(\sim 90 \%)$ exhibit a decrease in ID3 protein expression. ID3 inhibits TCFs by forming non-functional heterodimers and thereby prevents transcription of the target genes, e.g., TCF3, which in turn activates cyclin D3 and cyclin $\mathrm{E}^{32,46}$. TCF3 further regulates acinar cell identity by activating expression of the pancreatic transcription factor PTF $1 \mathrm{~A}^{33}$ and acinar transcription factor BHLHA15 (=Mist-1) ${ }^{47}$. A loss of ID3, therefore should lead to an overexpression of TCF3, resulting in an activation of the acinar cell program, whereas an activation of ID3 as shown by 
a
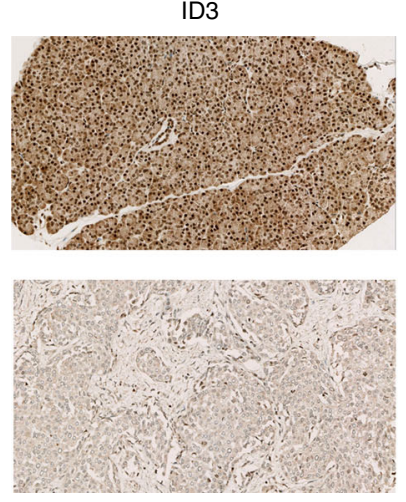

b

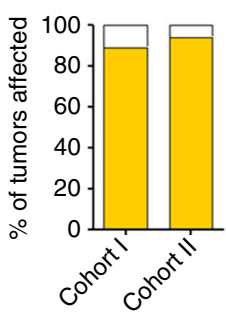

ARID1A
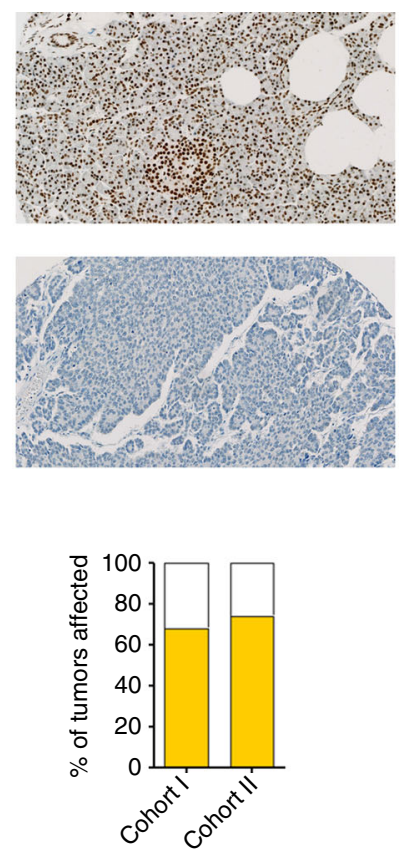

APC
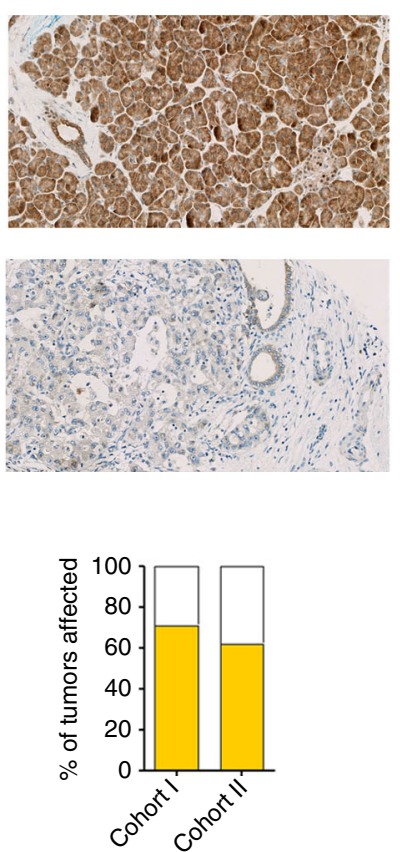

CDKN2A
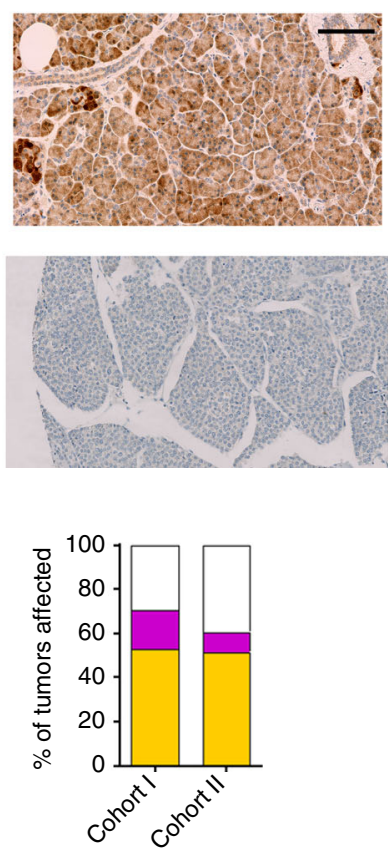

c

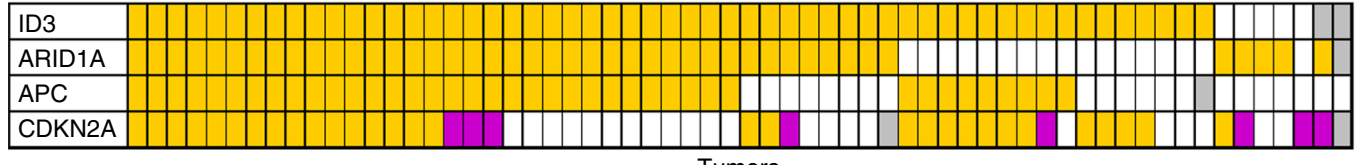

Tumors

\begin{tabular}{|l|l|}
\hline Gene deletion \\
\hline Gene amplification \\
\hline Hypermethylation promoter \\
\hline & Protein downregulation \\
\hline $\mathbf{x}$ & Protein overexpression \\
\hline
\end{tabular}

d

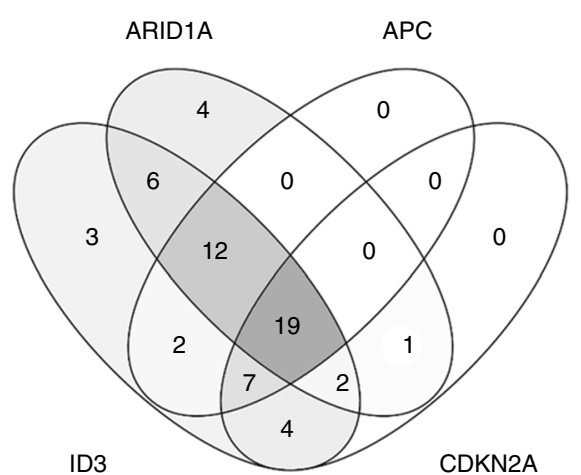

e

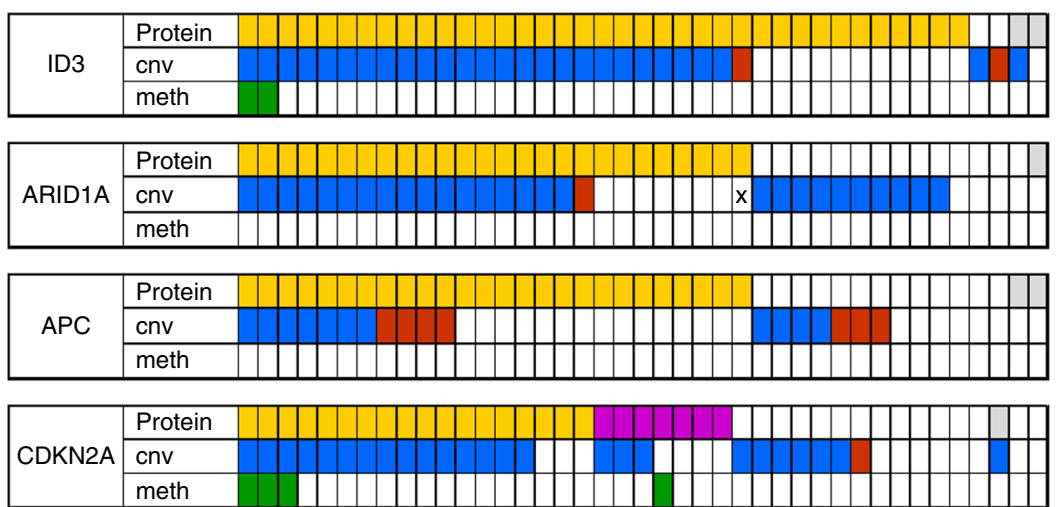

Fig. 5 ID3, ARID1A, APC, and CDKN2A are downregulated in the vast majority of ACC. a representative IHC for tumor and normal tissue; scale bar corresponds to $100 \mu \mathrm{m}$; all microscopy images are shown at the same magnification. $\mathbf{b}$ Percentages of altered tumors in cohort 1 and cohort 2 , a total of 69 tumors were investigated. c Overview of co-occurrence of protein alterations. $\mathbf{d}$ Venn diagram of co-occurrence of protein alterations. e IHC in combination with 450K data: co-occurrence of (epi-)genetic and protein alterations, grey: no data available

Kim et al. ${ }^{47}$ leads to an inactivation of the acinar cell program in PDAC. This gives rise to the hypothesis that ID3 expression might be the switch that distinguishes PDAC from ACC.

Our molecular characterization of ACC offers therapeutic targets for drugs currently tested in clinical trials for other tumors entities or that are already EMA-approved and/or FDA-approved (Supplementary Data File 10). This opens the opportunity that ACC patients can be included in basket trials, in which not the organ of the cancer but the molecular alterations are defining the inclusion criteria ${ }^{48}$. For instance, alterations in CDKN2A, ID3, and APC can be exploited by molecules interfering with the cell cycle regulator, $\mathrm{CDK} 4 / 6^{49}$. APC-altered tumors might additionally benefit from inhibiting one of the Wnt pathway members ${ }^{50}$. And in tumors lacking ARID1A, PI3K as well as ARID1B, EZH2 and PARP inhibition might lead to effective therapies ${ }^{51-55}$ (Supplementary Data File 10).

In summary, our study revealed that ACC are characterized by numerous copy number alterations and aberrantly methylated sites, and display distinct mutational signatures. Our analysis identified the four tumor suppressor genes ID3, ARID1A, APC, and CDKN2A as recurrently affected in ACC. The latter three have been reported as driver 


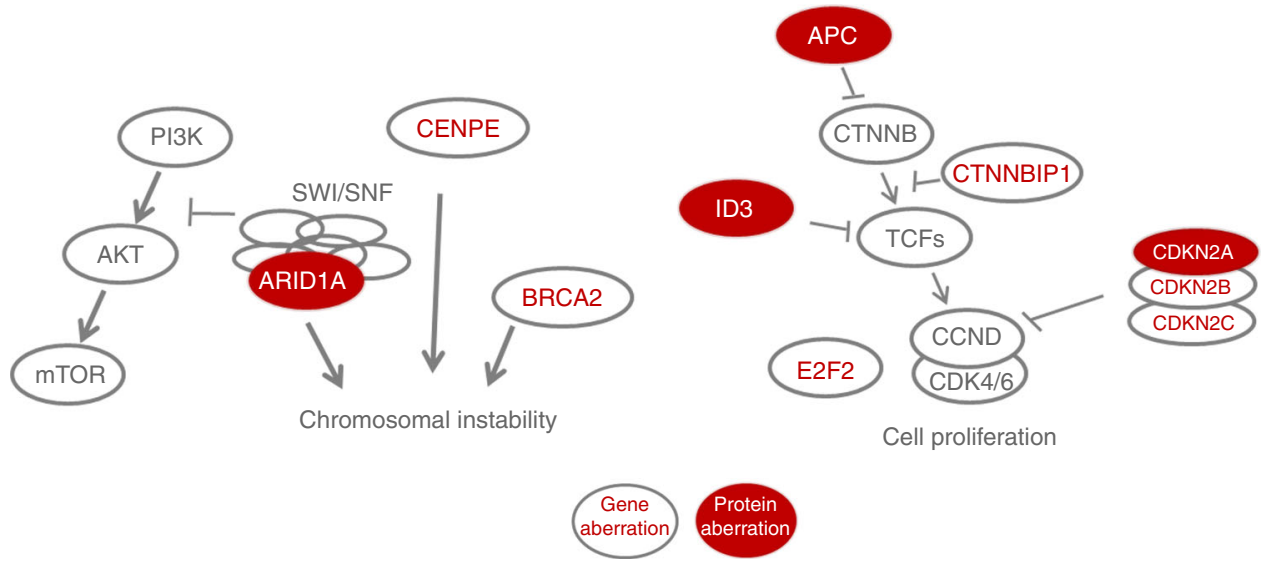

Fig. 6 Analyses reveal aberrations in genome stability and cell cycle control in ACC

genes in tumorigenesis ${ }^{26}$, therefore aberrations of these genes have to be considered for the development and progression of this disease. Moreover, these findings might offer insights for the development of treatment options for ACC.

\section{Methods}

Study cohorts. Two independent ACC cohorts were investigated (for details refer to Supplementary Table 1). Cohort 1 is a collection of 34 tumors (22 primary tumors and 12 metastases) and 24 normal pancreatic tissues, which were obtained from the Institute of Pathology, University of Heidelberg, Germany. Primary tumors from this cohort were used as discovery cohort, as matched germline controls were available for sequencing. Study cohort 2 was used as validation cohort and consists of 39 tumors (38 primary tumors and 1 metastasis) and 10 normal pancreatic tissues, which were obtained from the Consultation Center for Pancreatic and Endocrine Tumors of the Department of Pathology, Technical University Munich, Germany. Twenty and thirty-eight primary tumors and 3 and 1 metastases from cohort 1 and 2, respectively, and 8 normal pancreatic samples were further combined on a tissue microarray to facilitate immunohistochemical stainings. Most tissues were available as formalin fixed and paraffin embedded (FFPE) tissues, for five tumors fresh-frozen material was available. In addition, DNA from 17 pancreatic PNET obtained from FFPE tissue were investigated. Tumors were microdissected by a pathologist with a tumor purity of $>90 \%$. Tissue samples were provided by the tissue bank of the National Center for Tumor Diseases (NCT, Heidelberg, Germany) in accordance with the regulations of the tissue bank and the approval of the ethics committee of Heidelberg University (No. 207/2005; informed consent was obtained from all participants). The results shown on PDAC are based upon data generated by the TCGA Research Network: http:// cancergenome.nih.gov.

Conduct of Infinium HumanMethylation450 BeadChip array. DNA was isolated from FFPE tissue using the Invisorb Genomic DNA Kit II (Stratek, Birkenfeld, Germany). Quality was evaluated by real-time PCR analysis on Light Cycler 480 Real-Time PCR System (Roche, Mannheim, Germany) using the Infinium HD FFPE QC Kit (Illumina, San Diego, USA). DNA samples were bisulfite treated with the EZ-96 DNA Methylation Kit (Zymo Research Corporation, Orange, USA) and subsequently treated with the Infinium HD DNA Restoration Kit (Illumina). Each sample was whole genome amplified and enzymatically fragmented following the instructions in the Infinium HD FFPE Methylation Guide. The DNA was applied to the $450 \mathrm{~K}$ array (Illumina) and hybridization was performed for $16-24 \mathrm{~h}$ at $48{ }^{\circ} \mathrm{C}$. Microarray scanning was done using an iScan array scanner (Illumina).

Analysis of genome-wide DNA methylation via 450K. Analysis was run with the R Package RnBeads ${ }^{56}$. In summary, data were subjected to quality control, normalization with $\mathrm{BMIQ}^{57}$, and differential methylation was then called between groups using RnBeads's rank cutoff, which implements the difference in mean methylation, the quotient in mean methylation and the $p$-value obtained by limma test (for multiple comparisons) or Student's $t$-test (for paired analysis). Region annotations were implemented to analyze the average differential methylation in the following regions: gene bodies (defined by Ensembl ${ }^{58}$ ), promoter regions defined by RnBeads ${ }^{56}$ (regions spanning 1.5 kilo base pairs (kb) upstream and $0.5 \mathrm{~kb}$ downstream of the transcription start site for every gen $\mathrm{e}^{56}$ ), CpG Islands (defined according to the definition of the UCSC browser ${ }^{59}$ ), and promoter segments (intersection of promoters and $\mathrm{CpG}$ islands). For investigation of recurring methylation changes from primary tumors to metastasis, paired $t$-test was used to calculate differences. For cell type contributions, the method published by
Houseman et al. ${ }^{22}$ was used, which is a method similar to regression calibration. $450 \mathrm{~K}$ data from normal hematopoietic cell types were obtained from Reinius et al. ${ }^{60}$ In addition to the blood sample methylation data from this work, we added DNA methylation profiles from sorted pancreatic cells (acinar, duct, alpha, and beta cells). Phylogenetic trees were generated calculating Euclidean distance matrices based on all CpG sites on the array by the minimal evolution method ${ }^{61}$ using the fastme.bal function from the $\mathrm{R}$ package ape $\mathrm{e}^{62}$.

Analysis of genome-wide copy number via 450K. The Bioconductor package conumee $^{63}$ was used to calculate copy number alterations from the intensities obtained from the $450 \mathrm{~K}$ array (bin probe size was set to 5 , rest of parameters set to default). Gistic ${ }^{64}$ was then employed to investigate frequently deleted/amplified regions/genes (with default parameters).

Microsatellite instability analysis. Microsatellite instability was previously assessed in a subset of the here presented tumor samples in Bergmann et al. ${ }^{15}$ (also refer to Supplementary Data File 1), and analyses were conducted using the marker panels CAT25, BAT25, and BAT26 as developed by Findeisen et al. ${ }^{65}$ In brief, tumors were classified as high-level microsatellite instable (MSI), if at least two of the three markers displayed instability. PCR primers for the amplification of CAT25 were, forward 5V-CCTAGAAACCTTTATCCCTGCTT-3V, and reverse 5V-GAGCTTGCAGTGAGCTGAGA-3V. PCR primers were labeled at the 5Vend with FITC (BAT26 and CAT25) or HEX fluorescent dye (BAT25), respectively. Multiplex PCR was carried out in a total reaction volume of $25 \mu \mathrm{l}$ using a final concentration of $200 \mu \mathrm{mol}^{-1}$ deoxynucleotide triphosphates, $12.5 \mathrm{pmol} \mathrm{l}^{-1}$ of each primer, $1 \times$ PCR buffer $\left(20 \mathrm{mmol}^{-1}\right.$ Tris- $\left.\mathrm{HCl}(\mathrm{pH} 8.4), 50 \mathrm{mmol} \mathrm{l}^{-1} \mathrm{KCl}\right)$, $1.5 \mathrm{mmoll}^{-1} \mathrm{MgCl}_{2}$, and $0.75 \mathrm{U}$ of Taq DNA polymerase (Life Technologies/BRL, Eggenstein, Germany). Genomic DNA (50 ng) was used as a template. Reaction mixes were subjected to the following conditions: initial denaturation at $94^{\circ} \mathrm{C}$ for $5 \mathrm{~min}$ followed by 38 cycles of denaturation at $94^{\circ} \mathrm{C}$ for $30 \mathrm{~s}$, annealing at $55^{\circ} \mathrm{C}$ for $30 \mathrm{~s}$, extension at $72{ }^{\circ} \mathrm{C}$ for $30 \mathrm{~s}$, and a final extension step at $72{ }^{\circ} \mathrm{C}$ for $7 \mathrm{~min}$.

Whole-exome sequencing. Fresh frozen samples were checked and selected with H\&E stainings for high tumor cellularity. For DNA from fresh-frozen samples, library preparation was performed according to Agilent's "SureSelectXT Target Enrichment System for Illumina Paired-End Multiplexed Sequencing Library" kit, whereas for DNA from FFPE tissue the "SureSelect Automated Library Prep and Capture System SureSelectXT Automated Target Enrichment for Illumina PairedEnd Multiplexed Sequencing" kit was used. Samples were run paired-end (125 bp) on a HiSeq $2000 \mathrm{v} 4$. Alignment of data were processed by the following parameters: reference genome: hs37d5, alignment program: bwa- 0.7 .8 mem, alignment parameter: -T 0, duplication marking program: picard-1.125, default duplication marking program parameters were used (https://broadinstitute.github.io/picard/ command-line-overview.html\#MarkDuplicates). Alterations that are likely to be benign, so called FLAGS ${ }^{66}$ were excluded. Previously published WES studies of $\mathrm{ACC}^{13}, 14$ were utilized as a comparison to the here generated WES results. MutSigCV was used to calculate significantly recurrent mutations ${ }^{67}$.

Integrative analysis. To integrate changes in methylation and in copy numbers within the genes, data were categorized into the following nine groups: deleted and promoter hypermethylated, deleted only, promoter hypermethylated only, deleted and promoter hypomethylated, unaltered, amplified and promoter hypermethylated, promoter hypomethylated only, amplified only, and amplified and promoter hypomethylated. The following cutoffs were used: for copy number alterations the deletion and amplification thresholds from Gistic were employed. For differential methylation, a tumor was called to be hypo-/hypermethylated at a gene if the 
promoter methylation was below or above $20 \%$ of the mean methylation level of all normal pancreatic tissues.

Identification of cancer-related aberrations. Significantly differentially methylated genes and genes altered on the copy number levels were overlapped with multiple previously published gene lists in order to obtain a list as comprehensive as possible of cancer related genes (known cancer genes, candidate cancer genes, and oncomirs from King's college (http://ncg.kcl.ac.uk/download.php); ${ }^{28}$ tumor suppressors from Vanderbuilt (https://bioinfo.uth.edu/TSGene/Human_TSGs.txt); ${ }^{27}$ driver genes mutated, driver genes CNA, and cancer predisposition genes from Vogelstein et al.; ${ }^{26}$ epigenetic regulators from Plass et al. ${ }^{25}$ ).

Circos plots. The $\mathrm{R}$ package circlize was used to create circos plots. For copy number aberrations the total number of tumors which are amplified (red) or deleted (blue) out of all 41 primary tumors that were subjected to $450 \mathrm{~K}$ analysis is depicted. For methylation the difference of the promoter region means (tumor minus normal tissue) is depicted (hypermethylation dark green, hypomethylation yellow).

GO enrichment. For gene enrichment analysis the function annotation tool from the DAVID website was used ${ }^{68}$.

Immunohistochemical staining. Immunohistochemical analyses were performed using an automated slide staining system (Ventana BenchMark Ultra, Roche) and the avidin-biotin complex method ${ }^{15}$. All information on antibodies, such as source, catalogue and lot numbers, concentrations etc., is listed in Supplementary Data File 11. The secondary antibody was integrated in the used staining kit (Ventana Optiview DAB; Roche). Pretreatment was performed using the buffer ULTRA Cell Conditioning Solution CC1 (Roche), as listed in Supplementary Data File 11. Staining intensities were evaluated by a pathologist. Proteins were considered downregulated when the value of the tumor was at least one staining intensity lower than the lowest staining intensity of the normal tissues, whereas proteins were considered upregulated when the value of the tumor was at least one staining intensity higher than the highest staining intensity of the normal tissues.

Mutational signatures. Mutational frequencies were plotted using the SomaticSignatures package (version 2.8.4) available on Bioconductor ${ }^{69}$. WES data from ACC and publicly available WES from TCGA (available in the SomaticCancerAlterations Bioconductor package 1.8.2) were used to assess frequency of point mutations in the context of three nucleotides $(\mathrm{X}-\mathrm{A} / \mathrm{T}-\mathrm{X})^{70}$. For investigating the occurrence of published mutational signatures summarized by COSMIC (http:// cancer.sanger.ac.uk/cosmic/signatures) in ACC, we employed deconstructSigs $(\text { version } 1.8 .0)^{18}$.

Isolation of pure pancreatic cells. Pancreatic cells were isolated by FACS with antibodies specific for duct, acinar, endocrine alpha, and endocrine beta cells ${ }^{71}$. In brief, pancreatic cells were dispersed using trypsin $\left(10 \mathrm{~min}\right.$ at $\left.37^{\circ} \mathrm{C}\right)$ and occasional mechanical disruption with a p1000 micropipette. Cells were strained $(40 \mu \mathrm{m})$, washed, resuspended, and incubated with the appropriate 1:50 diluted primary antibodies (Alpha cells: Pal Antibody (DHIC2-2C12), pan-islet cells: HPi2 Antibody (HIC1-2B4.2B), acinar cells: HPxl Antibody (HIC0-3B3), and duct cells: HPd1 Antibody (DHIC2-4A10) from Novus Biologicals (catalogue\# NBP1-18949, NBP1-18946, NBP1-18951, and NBP1-18953). Secondary antibodies (PE-conjugated anti-mouse IgM ( $\mu$ chain) and Dylight488-conjugated anti-mouse IgG [ $1+$ $2 \mathrm{a}+3$ ]; Jackson ImmunoResearch, catalog\# 115-545-164 and 115-116-075) were added at 1:200 and cells were subsequently sorted with an inFlux V-GS (BD Biosciences) equipped with a $100 \mu \mathrm{m}$ nozzle. Duct and acinar cell $450 \mathrm{~K}$ data from Lehmann-Werman et al. ${ }^{36}$ was used and endocrine $\alpha$-cell and $\beta$-cell $450 \mathrm{~K}$ data were additionally generated.

Data availability. The methylation array and exome-sequencing data of all generated datasets are deposited at the European Genome-Phenome Archive under study accession number EGAS00001002533. Data for PDAC were downloaded from the TCGA portal (https://portal.gdc.cancer.gov/projects/TCGA-PAAD). All other relevant data are available from the authors.

Received: 2 March 2017 Accepted: 18 August 2017

Published online: 06 November 2017

\section{References}

1. Klimstra, D. H. R., Klöppel, G., Morohoshi, T. \& Ohike, N. in WHO Classification of Tumours of the Digestive System (eds Bosman, F. T. et al.) 314-318 (International Agency for Research on Cancer, Lyon, 2010).

2. Lowery, M. A. et al. Acinar cell carcinoma of the pancreas: new genetic and treatment insights into a rare malignancy. Oncologist 16, 1714-1720 (2011).
3. Wisnoski, N. C., Townsend, C. M. Jr, Nealon, W. H., Freeman, J. L. \& Riall, T. S 672 patients with acinar cell carcinoma of the pancreas: a population-based comparison to pancreatic adenocarcinoma. Surgery 144, 141-148 (2008).

4. Wood, L. D. \& Klimstra, D. S. Pathology and genetics of pancreatic neoplasms with acinar differentiation. Semin. Diagn. Pathol. 31, 491-497 (2014).

5. Hartwig, W. et al. Acinar cell carcinoma of the pancreas: is resection justified even in limited metastatic disease? Am. J. Surg. 202, 23-27 (2011).

6. Biankin, A. V. et al. Pancreatic cancer genomes reveal aberrations in axon guidance pathway genes. Nature 491, 399-405 (2012).

7. Waddell, N. et al. Whole genomes redefine the mutational landscape of pancreatic cancer. Nature 518, 495-501 (2015).

8. Scarpa, A. et al. Whole-genome landscape of pancreatic neuroendocrine tumours. Nature 543, 65-71 (2017)

9. Abraham, S. C. et al. Genetic and immunohistochemical analysis of pancreatic acinar cell carcinoma: frequent allelic loss on chromosome 11p and alterations in the APC/beta-catenin pathway. Am. J. Pathol. 160, 953-962 (2002).

10. Hoorens, A. et al. Pancreatic acinar cell carcinoma. An analysis of cell lineage markers, p53 expression, and Ki-ras mutation. Am. J. Pathol. 143, 685-698 (1993).

11. de Wilde, R. F. et al. Analysis of LKB1 mutations and other molecular alterations in pancreatic acinar cell carcinoma. Mod. Pathol. 24, 1229-1236 (2011).

12. Furlan, D. et al. APC alterations are frequently involved in the pathogenesis of acinar cell carcinoma of the pancreas, mainly through gene loss and promoter hypermethylation. Virchows Arch. 464, 553-564 (2014).

13. Furukawa, T. et al. Whole exome sequencing reveals recurrent mutations in BRCA2 and FAT genes in acinar cell carcinomas of the pancreas. Sci. Rep. 5, 8829 (2015).

14. Jiao, Y. et al. Whole-exome sequencing of pancreatic neoplasms with acinar differentiation. J. Pathol. 232, 428-435 (2014).

15. Bergmann, F. et al. Acinar cell carcinomas of the pancreas: a molecular analysis in a series of 57 cases. Virchows Arch. 465, 661-672 (2014).

16. La Rosa, S. et al. Clinicopathologic study of 62 acinar cell carcinomas of the pancreas: insights into the morphology and immunophenotype and search for prognostic markers. Am. J. Surg. Pathol. 36, 1782-1795 (2012).

17. Alexandrov, L. B. et al. Signatures of mutational processes in human cancer. Nature 500, 415-421 (2013).

18. Rosenthal, R., McGranahan, N., Herrero, J., Taylor, B. S. \& Swanton, C. DeconstructSigs: delineating mutational processes in single tumors distinguishes DNA repair deficiencies and patterns of carcinoma evolution. Genome Biol. 17, 31 (2016).

19. Alexandrov, L. B. et al. Mutational signatures associated with tobacco smoking in human cancer. Science 354, 618-622 (2016).

20. Aran, D., Sirota, M. \& Butte, A. J. Systematic pan-cancer analysis of tumour purity. Nat. Commun. 6, 8971 (2015).

21. Cancer Genome Atlas Research Network. et al. The Cancer Genome Atlas PanCancer analysis project. Nat. Genet. 45, 1113-1120 (2013).

22. Houseman, E. A. et al. DNA methylation arrays as surrogate measures of cell mixture distribution. BMC Bioinformatics 13, 86 (2012).

23. Morris, T. J. et al. ChAMP: 450k chip analysis methylation pipeline. Bioinformatics 30, 428-430 (2014).

24. Makohon-Moore, A. P. et al. Limited heterogeneity of known driver gene mutations among the metastases of individual patients with pancreatic cancer. Nat. Genet. 49, 358-366 (2017).

25. Plass, C. et al. Mutations in regulators of the epigenome and their connections to global chromatin patterns in cancer. Nat. Rev. Genet. 14, 765-780 (2013).

26. Vogelstein, B. et al. Cancer genome landscapes. Science 339, 1546-1558 (2013)

27. Zhao, M., Kim, P., Mitra, R., Zhao, J. \& Zhao, Z. TSGene 2.0: an updated literature-based knowledgebase for tumor suppressor genes. Nucleic Acids Res. 44, D1023-D1031 (2016)

28. An, O., Dall'Olio, G. M., Mourikis, T. P. \& Ciccarelli, F. D. NCG 5.0: updates of a manually curated repository of cancer genes and associated properties from cancer mutational screenings. Nucleic Acids Res. 44, D992-D999 (2016).

29. Kleeff, J. et al. Pancreatic cancer. Nat. Rev. Dis. Primers 2, 16022 (2016).

30. Franko, J. et al. Loss of heterozygosity predicts poor survival after resection of pancreatic adenocarcinoma. J. Gastrointest. Surg. 12, 1664-1672 (2008).

31. Bailey, P. et al. Genomic analyses identify molecular subtypes of pancreatic cancer. Nature 531, 47-52 (2016).

32. Lee, S. H. et al. The Id3/E47 axis mediates cell-cycle control in human pancreatic ducts and adenocarcinoma. Mol. Cancer Res. 9, 782-790 (2011).

33. Dufresne, M. et al. Id3 modulates cellular localization of bHLH Ptf1-p48 protein. Int. J. Cancer 129, 295-306 (2011)

34. Oakes, C. C. et al. Evolution of DNA methylation is linked to genetic aberrations in chronic lymphocytic leukemia. Cancer Discov. 4, 348-361 (2014)

35. Sproul, D. et al. Tissue of origin determines cancer-associated CpG island promoter hypermethylation patterns. Genome Biol. 13, R84 (2012). 
36. Lehmann-Werman, R. et al. Identification of tissue-specific cell death using methylation patterns of circulating DNA. Proc. Natl Acad. Sci. USA 113 E1826-E1834 (2016).

37. Morohoshi, T., Held, G. \& Kloppel, G. Exocrine pancreatic tumours and their histological classification. A study based on 167 autopsy and 97 surgical cases. Histopathology 7, 645-661 (1983).

38. Clark, C. E. et al. Dynamics of the immune reaction to pancreatic cancer from inception to invasion. Cancer Res. 67, 9518-9527 (2007).

39. Feber, A. et al. Using high-density DNA methylation arrays to profile copy number alterations. Genome Biol. 15, R30 (2014).

40. Watanabe, R. et al. SWI/SNF factors required for cellular resistance to DNA damage include ARID1A and ARID1B and show interdependent protein stability. Cancer Res. 74, 2465-2475 (2014).

41. Dykhuizen, E. C. et al. BAF complexes facilitate decatenation of DNA by topoisomerase IIalpha. Nature 497, 624-627 (2013).

42. Schmitt-Graeff, A. et al. Coordinated expression of cyclin D1 and LEF-1/TCF transcription factor is restricted to a subset of hepatocellular carcinoma. Liver Int. 25, 839-847 (2005).

43. Klein, E. A. \& Assoian, R. K. Transcriptional regulation of the cyclin D1 gene at a glance. J. Cell Sci. 121, 3853-3857 (2008).

44. Moore, P. S. et al. Pancreatic tumours: molecular pathways implicated in ductal cancer are involved in ampullary but not in exocrine nonductal or endocrine tumorigenesis. Br. J. Cancer 84, 253-262 (2001).

45. Romagosa, C. et al. p16(Ink4a) overexpression in cancer: a tumor suppressor gene associated with senescence and high-grade tumors. Oncogene $\mathbf{3 0}$ 2087-2097 (2011).

46. Schmitz, R. et al. Burkitt lymphoma pathogenesis and therapeutic targets from structural and functional genomics. Nature 490, 116-120 (2012).

47. Kim, S. et al. The basic helix-loop-helix transcription factor E47 reprograms human pancreatic cancer cells to a quiescent acinar state with reduced tumorigenic potential. Pancreas 44, 718-727 (2015).

48. Ornes, S. Core concept: basket trial approach capitalizes on the molecular mechanisms of tumors. Proc. Natl Acad. Sci. USA 113, 7007-7008 (2016).

49. O'Leary, B., Finn, R. S. \& Turner, N. C. Treating cancer with selective CDK4/6 inhibitors. Nat. Rev. Clin. Oncol. 13, 417-430 (2016).

50. Kahn, M. Can we safely target the WNT pathway? Nat. Rev. Drug Discov. 13, 513-532 (2014).

51. Samartzis, E. P. et al. Loss of ARID1A expression sensitizes cancer cells to PI3K- and AKT-inhibition. Oncotarget 5, 5295-5303 (2014).

52. Shen, J. et al. ARID1A deficiency impairs the DNA damage checkpoint and sensitizes cells to PARP inhibitors. Cancer Discov. 5, 752-767 (2015).

53. Helming, K. C. et al. ARID1B is a specific vulnerability in ARID1A-mutant cancers. Nat. Med. 20, 251-254 (2014).

54. Bitler, B. G. et al. Synthetic lethality by targeting EZH2 methyltransferase activity in ARID1A-mutated cancers. Nat. Med. 21, 231-238 (2015).

55. Williamson, C. T. et al. ATR inhibitors as a synthetic lethal therapy for tumours deficient in ARID1A. Nat. Commun. 7, 13837 (2016).

56. Assenov, Y. et al. Comprehensive analysis of DNA methylation data with RnBeads. Nat. Methods 11, 1138-1140 (2014).

57. Teschendorff, A. E. et al. A beta-mixture quantile normalization method for correcting probe design bias in Illumina Infinium $450 \mathrm{k}$ DNA methylation data. Bioinformatics 29, 189-196 (2013).

58. Ensemble 73. http://sep2013.archive.ensembl.org.

59. CpG Island Track UCSC Browser. https://genome.ucsc.edu/cgi-bin/hgTrackUi? hgsid=383138943_cuVjAzBaxUkx2fiCOkIfa81Sjr6U\&c=chr21\&g=cpgIslandSuper\&cpgIslandSuper $=$ show.

60. Reinius, L. E. et al. Differential DNA methylation in purified human blood cells: implications for cell lineage and studies on disease susceptibility. PLOS ONE 7, e41361 (2012).

61. Desper, R. \& Gascuel, O. Fast and accurate phylogeny reconstruction algorithms based on the minimum-evolution principle. J. Comput. Biol. 9, 687-705 (2002)

62. Paradis, E., Claude, J. \& Strimmer, K. APE: analyses of phylogenetics and evolution in R language. Bioinformatics 20, 289-290 (2004).

63. Hovestadt, V. \& Zapatka, M. Conumee: Enhanced copy-number variation analysis using Illumina 450k methylation arrays. R Package Version 0.99 .4 http://www.bioconductor.org/packages/release/bioc/html/conumee.html. (2016).
64. Mermel, C. H. et al. GISTIC2.0 facilitates sensitive and confident localization of the targets of focal somatic copy-number alteration in human cancers. Genome Biol. 12, R41 (2011).

65. Findeisen, P. et al. T25 repeat in the 3' untranslated region of the CASP2 gene: a sensitive and specific marker for microsatellite instability in colorectal cancer. Cancer Res. 65, 8072-8078 (2005).

66. Shyr, C. et al. FLAGS, frequently mutated genes in public exomes. BMC Med. Genomics 7, 64 (2014).

67. Lawrence, M. S. et al. Mutational heterogeneity in cancer and the search for new cancer-associated genes. Nature 499, 214-218 (2013).

68. Huang da, W., Sherman, B. T. \& Lempicki, R. A. Systematic and integrative analysis of large gene lists using DAVID bioinformatics resources. Nat. Protoc 4, 44-57 (2009).

69. Gehring, J. S., Fischer, B., Lawrence, M. \& Huber, W. SomaticSignatures: inferring mutational signatures from single-nucleotide variants. Bioinformatics 31, 3673-3675 (2015).

70. Gehring, J. SomaticCancerAlterations: Somatic Cancer Alterations. R Package Version 1.8.2. https://bioconductor.org/packages/release/data/experiment/ $\mathrm{html} /$ SomaticCancerAlterations.html (2016).

71. Dorrell, C. et al. Transcriptomes of the major human pancreatic cell types. Diabetologia 54, 2832-2844 (2011).

\section{Acknowledgements}

We thank the microarray unit and the high-throughput sequencing unit of the DKFZ Genomics and Proteomics Core Facility for providing the Illumina HumanMethylation 450 arrays, performing whole-exome sequencing and related services. We thank the Data Management and Genomics IT from the eilslabs for aligning sequencing data and calling SNVs. Funding for this study comes in part from the German Cancer Consortium (DKTK) and the Helmholtz Foundation.

\section{Author contributions}

C.J., F.B., C.P., O.P., and P.S. designed and supervised the study. C.J., F.B., and D.v.d.D. performed experiments. C.J., R.T., and Y.A. analyzed data. F.B., O.S., T.H., G.K., and P.Schi. acquired and processed ACC patient tissues, acquired and annotated clinical and follow-up data. C.D., M.G., J.M., and Y.D. generated data for sorted pancreatic cells. C.J., O.P., and P.S. wrote the manuscript. F.B., R.T., C.P., O.P., and P.S. gave conceptual advice. All authors interpreted the results and implications and contributed to the final manuscript.

\section{Additional information}

Supplementary Information accompanies this paper at doi:10.1038/s41467-017-01118-x.

Competing interests: The authors declare no competing financial interests.

Reprints and permission information is available online at http://npg.nature.com/ reprintsandpermissions/

Publisher's note: Springer Nature remains neutral with regard to jurisdictional claims in published maps and institutional affiliations.

Open Access This article is licensed under a Creative Commons Attribution 4.0 International License, which permits use, sharing, adaptation, distribution and reproduction in any medium or format, as long as you give appropriate credit to the original author(s) and the source, provide a link to the Creative Commons license, and indicate if changes were made. The images or other third party material in this article are included in the article's Creative Commons license, unless indicated otherwise in a credit line to the material. If material is not included in the article's Creative Commons license and your intended use is not permitted by statutory regulation or exceeds the permitted use, you will need to obtain permission directly from the copyright holder. To view a copy of this license, visit http://creativecommons.org/ licenses/by/4.0/

(C) The Author(s) 2017 